\title{
Detection of Human Viral RNA via a Combined Fluorescence and SERS Molecular Beacon Assay
}

\author{
Michael Y. Sha - Sharron Penn • \\ Griff Freeman - William E. Doering
}

Published online: 3 May 2007

(C) Humana Press Inc. 2007

\begin{abstract}
A dual-mode molecular beacon on a multiplexed substrate has been developed and applied to the measurement of unlabeled human viral RNA. The detection system is based on a combined surface-enhanced Raman scattering (SERS) and fluorescent molecular beacon assay that is assembled on Nanobarcodes ${ }^{\mathrm{TM}}$ particles. In this assay, a molecular beacon probe terminated with a fluorescent Raman label dye is conjugated to the metallic Nanobarcodes $^{\mathrm{TM}}$ particles. The molecular beacon probe is a single-stranded oligonucleotide that has been designed with a hairpin structure that holds the dye at $3^{\prime}$-end close to the particle surface when the probe is attached through a $5^{\prime}$ thiol group. In this configuration, the SERS spectrum of the label was obtained and its fluorescence quenched because the dye is in very close proximity to a noble metal surface with nanoscale features (Nanobarcodes ${ }^{\mathrm{TM}}$ particles). The SERS signal decreased and the fluorescence signal increased when target viral RNA was captured by this molecular beacon probe. In addition, a hepatitis $\mathrm{C}$ virus reverse transcriptasepolymerase chain reaction (HCV RT-PCR) product was detected using this dual-mode beacon. The development of a multiplexed, label-free assay system with the reassurance offered by detection of two distinctly separate signals offers significant benefits for rapid molecular diagnostics.
\end{abstract}

Keywords SERS beacon - label-free - molecular beacon . RNA detection

M. Y. Sha $(\varpi) \cdot S$. Penn $\cdot$ G. Freeman $(\bowtie) \cdot$ W. E. Doering Oxonica Inc, 665 Clyde Avenue, Suite A,

Mountain View, CA 94043, USA

e-mail: michael.sha@oxonica.com

e-mail: griff.freeman@oxonica.com

\section{Introduction}

The application of surface-enhanced Raman scattering (SERS) to the measurement of biochemically interesting species began shortly after the first description [1] and explanation [2] of the phenomenon. Cotton's study of cytochrome $c$ and myoglobin [3] helped set the stage for the recent explosion of work that includes the detection of anthrax [4], cancer diagnostics [5], a glucose biosensor [6], DNA and RNA detection [7-10], protein immunoassays [11], and cell labeling [12]. Several characteristics of SERS make it an attractive method for the detection of biomolecules. Because of the uniqueness of molecular Raman spectra, it is possible to detect several species simultaneously (multiplexed detection) [7, 13]. As Raman scattering (and SERS) is not inherently susceptible to photobleaching, extended exposures to optical excitation sources are possible without signal degradation [14]. The higher sensitivity and lower detection limit, due to the SERS enhancement and real-time response, are particularly useful for a quantitative assay, and the detection of single molecules by SERS indicates the potential for measurements with very high sensitivity [15-17].

Fluorescent molecular beacons were first developed in 1996 [18]. The molecular beacon is a single-stranded "loop-and-stem" DNA oligonucleotide carrying both a fluorochrome and a nonfluorescent quencher chromophore at opposite ends of the strand. In the absence of a complementary strand, the two ends are near each other due to intramolecular bonding. When the loop binds to a complementary nucleic acid target, the molecule changes its conformation removing the fluorochrome from the vicinity of the quencher and resulting in unquenched fluorescence. Quenching in the molecular beacon is typically achieved with the nonfluorescent chromophore DABCYL 
(4-((-4-(dimethylamino)-phenyl)-azo)-benzoic acid). However, this concept has also been adapted to metal nanoparticles where the metal surface acts to quench the fluorescence rather than an organic moiety [19-23]. Metals can efficiently quench emission from dyes located within a few nanometers of their surface [24]. For this work, we have used Nanobarcodes ${ }^{\mathrm{TM}}$ particles (NBC) as our metal surface. These particles have been shown to have a fluorescence quenching efficiency around $89-94 \%$ with the dyes TAMRA and Texas red [22] and $1.4 \mathrm{~nm} \mathrm{Au}$ colloid particles have demonstrated a quenching efficiency of $98-99 \%$ [19]. These results indicate that metal surfaces can function even better than a nonfluorescent chromophore such as DABCYL [19].

Wabuyele and Vo-Dinh [9] have described the application of SERS molecular sentinel nanoprobes conjugated to 35-50 nm Ag colloids for the detection of HIV-1. They demonstrated the feasibility of the SERS molecular sentinel nanoprobe for detection of HIV-1 DNA. They reported a SERS quenching efficiency of about $75 \%$ for the pure oligo target and $56 \%$ for its polymerase chain reaction (PCR) amplicon [9]. Faulds et al. reported a surface-enhanced resonance Raman scattering (SERRS) beacon which combine SERRS and fluorescence to detect DNA [10]. However, two dyes are required in this SERRS beacon, which could cause two different Raman spectra, making multiplexed assays difficult.

In this manuscript, we have combined the sensitivity of SERS technology and multiplexing potential of NBC to develop a SERS beacon assay for detection of viral RNA. A single NBC is about $300 \mathrm{~nm}$ wide and $6-9 \mu \mathrm{m}$ in length. NBC are synthesized by sequential electrochemical deposition of metal ions into templates with uniformly sized pores [25-27]. Coding of the 9- $\mu \mathrm{m}$ length with 11 stripes of two metals (silver and gold) could create 1,056 different striping patterns, giving a huge multiplexing capability. This concept has already been applied to multiplexed SNP genotyping [28] and a multiplexed fluorescence molecular beacon for viral RNA detection $[22,23]$. Our new dualmode design utilizes a molecular beacon probe with a hairpin structure, which holds the dye at the $3^{\prime}$ end close to the nanoparticle surface when the probe is assembled to it by a 5 -thiol group. Fluorescence from the dye is quenched when it is in proximity to the surface of metal nanoparticles such as NBC $[22,23]$. On the other hand, SERS spectra are observed when the dye is held near the surface of the metal (Ag or $\mathrm{Au}$ ).

When the probe hybridizes to its target sequence, the Raman label dye is forced to separate from the metal surface, and the SERS signal drops in intensity because the Raman enhancement $\left(E_{\mathrm{R}}\right)$ depends strongly on the distance $(d)$ between the Raman label and the metal nanoparticle $\left(E_{\mathrm{R}}=d^{-12}\right)[29,30]$. Meanwhile, the fluorescence increases

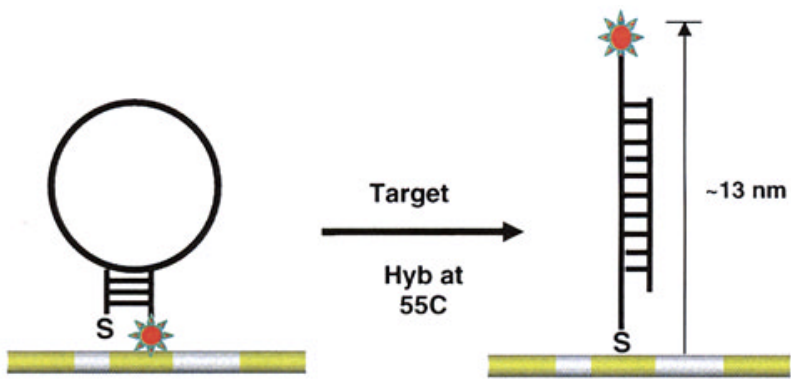

SERS On, Fluorescence Quenched

SERS Off \& Fluorescence On

Fig. 1 Cartoon of the SERS molecular beacon assay on a striped metal particle

as the dye is no longer efficiently quenched by the metal (Fig. 1). By applying this bioassay system, we could detect many oligonucleotide targets such as viral RNA or DNA, bio-threat agents, gene expression products, food-born pathogens, SNP or mutant detection, or even cancer biomarker proteins by using an aptamer as the detection probe.

\section{Materials and Methods}

\section{SERS Beacon Probe Design}

The stem-loop structures of the molecular beacons were designed using MFold software [31]. The HCV probe sequence was designed from the $5^{\prime}$ UTR region. The sequence was: $5^{\prime}$ thiol $\left(\mathrm{CH}_{2}\right) 6$ gegag CAT AGT GGT CTG CGG AAC CGG TGA ctcgc $\left(\mathrm{CH}_{2}\right) 7$ Cy5 -3'. The oligo target sequence was: TCA CCG GTT CCG CAG ACC ACT ATG. All probes and targets were purchased from BioSource (Camarillo, CA). The HCV viral RNA (Armored RNA ${ }^{\circledR}$ ) was purchased from Ambion Diagnostics (TX).

Nanobarcodes ${ }^{\mathrm{TM}}$ Synthesis

NBC were manufactured as previously described [25-27]. Briefly, alternating layers of gold and silver were electroplated into the pores of an alumina template. Then the template was dissolved using strong base, resulting in the release of the desired particles. The NBC used in this study were $250 \mathrm{~nm}$ by $6 \mu \mathrm{m}$ and contained six metallic segments.

\section{Probe Attachment Protocols}

Oligonucleotide probes were assembled onto the NBC as follows: Approximately $10^{8} \mathrm{NBC}$ in $100 \mu \mathrm{l}$ water were washed twice with $10 \mathrm{mM}$ phosphate-buffered saline (PBS) and resuspended in $100 \mu 110 \mathrm{mM}$ PBS. Next, $500 \mu \mathrm{l}$ of 
$5 \mu \mathrm{M}$ dye-functionalized oligonucleotide probes was added and allowed to self-assemble overnight at room temperature, with gentle rotation. After assembly, $600 \mu \mathrm{L}$ of $0.3 \mathrm{M}$ $\mathrm{NaCl}$ in $10 \mathrm{mM}$ PBS was added and allowed to react for a 2-h "aging" period [32]. The particles were then washed twice in $0.3 \mathrm{M} \mathrm{NaCl}$ containing $10 \mathrm{mM}$ PBS to remove unbound probe, resuspended in $100 \mu \mathrm{L} 10 \mathrm{mM}$ PBS and stored at $4^{\circ} \mathrm{C}$ until ready for use.

\section{Hybridization Assay}

Approximately $3 \times 10^{6}$ oligo-functionalized NBC in $3 \mu \mathrm{L}$ of PBS were added to $33 \mu \mathrm{L}$ of hybridization buffer (HS114, Molecular Research Center, Inc), oligo or PCR target in a volume of $16 \mu \mathrm{L}$ and boiled (for PCR sample) for $2 \mathrm{~min}$ to denature the DNA. The reaction mixtures were tumbled gently for $1 \mathrm{~h}$ at $55^{\circ} \mathrm{C}$. The reaction mixtures were then washed with $500 \mu \mathrm{L} 1 \times \mathrm{SSC}$ for $5 \mathrm{~min}$, followed by $500 \mu \mathrm{L} 0.1 \times \mathrm{SSC}$ for $5 \mathrm{~min}$.

\section{RT-PCR and Lambda Exonuclease Digestion}

Reactions were performed using the Superscript ${ }^{\mathrm{TM}}$ one-step reverse transcriptase-PCR (RT-PCR) kit (Invitrogen, CA). Five microliters viral RNA was incubated at $75^{\circ} \mathrm{C}$ for $3 \mathrm{~min}$ and added to a mixture containing $25 \mu \mathrm{L} 2 \times$ reaction buffer, $1 \mu \mathrm{L} 10 \mu \mathrm{M}$ primer $\# 1,1 \mu \mathrm{L} 10 \mu \mathrm{M}$ primer $\# 2,1 \mu \mathrm{L} \mathrm{Taq}$ polymerase, and $17 \mu \mathrm{l} \mathrm{H}_{2} \mathrm{O}$ (to $50 \mu \mathrm{l}$ total reaction volume). Multiplexed RT-PCR was performed using the same reagent concentrations. The following conditions were performed on a thermocycler: $50^{\circ} \mathrm{C} 30 \mathrm{~min}, 94^{\circ} \mathrm{C}$ $2 \mathrm{~min}$, then 40 cycles at $94^{\circ} \mathrm{C}, 15 \mathrm{~s} ; 60^{\circ} \mathrm{C} 30 \mathrm{~s}$; and $72^{\circ} \mathrm{C}$, $30 \mathrm{~s}$; and a final $72^{\circ} \mathrm{C} 10 \mathrm{~min}$ and hold at $4{ }^{\circ} \mathrm{C}$. The double stranded PCR product was designed with a $5^{\prime}$ phosphate group, such that lambda exonuclease could be used to digest away the phosphorylated $5^{\prime}$ strand, leaving the nonphosphorylated $3^{\prime}$ strand for hybridization to the molecular beacon probe. The reaction was allowed to proceed for $20 \mathrm{~min}$ at $37^{\circ} \mathrm{C}$ and then boiled for 1 min to inhibit any further enzyme activity. All PCR products were designed to locate the oligonucleotide's complementary sequence approximately in the middle of the amplicon. The lengths of the HCV PCR products were about 95 bases

\section{Data Collection and Analysis}

After hybridization, the particles were resuspended in $8 \mu \mathrm{L}$ of $5 \mathrm{mM}$ PBS and deposited on a quartz slide. Raman spectra were obtained using a Renishaw inVia Raman microscope (Renishaw Inc, UK) with $785 \mathrm{~nm}$ laser excitation, a $5 \times$ objective, $1 \mathrm{~s}$ accumulation time and with the spectrometer grating centered at $1,300 \mathrm{~cm}^{-1}$. Data were analyzed using custom, in-house software. Fluorescence data were acquired by transferring the sample into a glassbottom 96-well plate and imaging with a Zeiss Axiovert 100 microscope fitted with a Prior H107 stage, Sutter Instruments $300 \mathrm{~W}$ Xe lamp with liquid light guide, Physik Instrument 400 micron travel objective positioner and Photometrics CoolSnapHQ camera. Images were acquired with a $63 \times / 1.4 \mathrm{NA}$ oil objective. The microscope and all components were controlled by a proprietary software package that performs intra- and inter-well moves, automatically focuses at each new position, acquires a reflectance image of the particles at $405 \mathrm{~nm}$, and finally acquires the corresponding fluorescence image. The reflectance and fluorescence image pairs were analyzed by $\mathrm{NBSee}^{\mathrm{TM}}$ Software, a proprietary image analysis software package that identifies the NBC and quantifies their associated fluorescence.

\section{Results and Discussion}

To generate a SERS signal from an oligonucleotide probe attached to the NBC surface, we carefully designed an $\mathrm{HCV}$ probe with stem and loop sequence that allowed the formation of a hairpin structure to hold the Raman label dye in close proximity to the metal surface (Fig. 2). The probe sequence was selected from the HCV-la $5^{\text {' UTR region }}$ (Fig. 2a). By applying the Mfold software, we were able to select a sequence which has a loop and stem secondary structure under the hybridization conditions that we used (Fig. 2b). We assembled the HCV molecular beacon probe with the NBC code 101010 (1 indicates Ag and 0 indicates $\mathrm{Au}$ ) overnight as described in the Materials and Methods section. After washing two times with buffer, we deposited $3 \mu \mathrm{L} \mathrm{NBC}$-probes onto the quartz slide and acquired the SERS with a Renishaw inVia Raman microscope at $785-\mathrm{nm}$ excitation.

As shown in Fig. 3a, Cy5-terminated HCV oligo probes gave a strong SERS spectrum when assembled on NBC 101010. To confirm that the observed SERS spectrum was actually from Cy5, we added $5 \mu \mathrm{L}$ of $1 \mu \mathrm{M}$ Cy 5 Mono NHS Ester (Amersham Bioscience s) into $10 \mu \mathrm{L}$ NBC 101010 , and it was incubated $10 \mathrm{~min}$. The resulting spectrum showed that the SERS obtained from pure Cy5 (no oligo) was similar to the Cy5-HCV probe (Fig. 3b vs. 3a). Moreover, we've obtained a similar SERS fingerprint using $50 \mathrm{~nm}$ gold nanoparticles (data not shown), and others have shown comparable data using $25 \mathrm{~nm}$ gold cores upon which silver was grown [7]. Furthermore, we have demonstrated that the degree of silver or gold stripes components on NBC do not significantly affect the SERS spectrum, which allows multiplexing applications based on the NBC striping patterns (Fig. 3c,d). 
Fig. 2 HCV molecular beacon probe design and secondary structure. a $\mathrm{HCV}$ molecular beacon probe (red line) and RT-PCR primers pair HCV5p and HCV3p was designed according HCV 5' UTR region. b HCV molecular beacon probe secondary structure was calculated under $0.1 \mathrm{M} \mathrm{NaCl}$ at 55C condition by Mfold software. c HCV RT-PCR amplicon in $1.2 \%$ agarose gel electrophoresis. Well line $l$ for standard 25 bp DNA ladder and line 2 shows HCV amplicon with the right size ( $95 \mathrm{bp}$ )

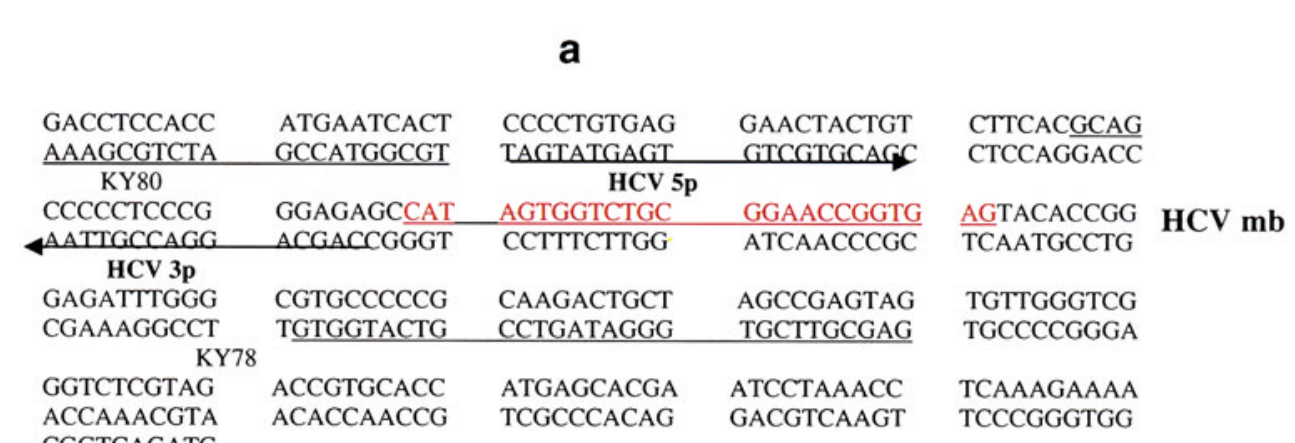

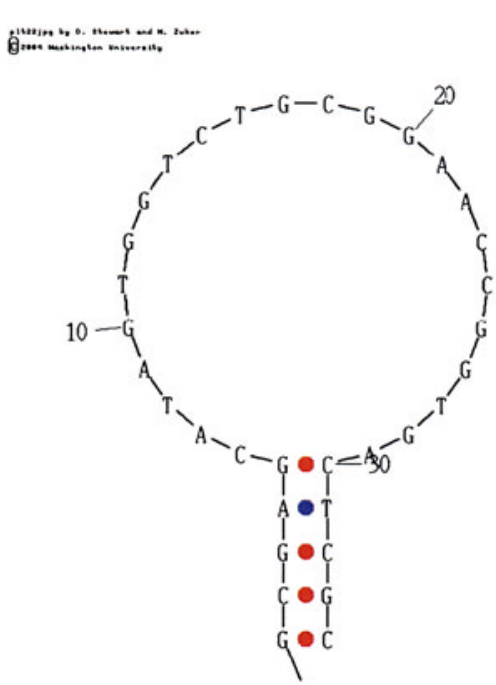

$\mathrm{dG}=0.9$

b

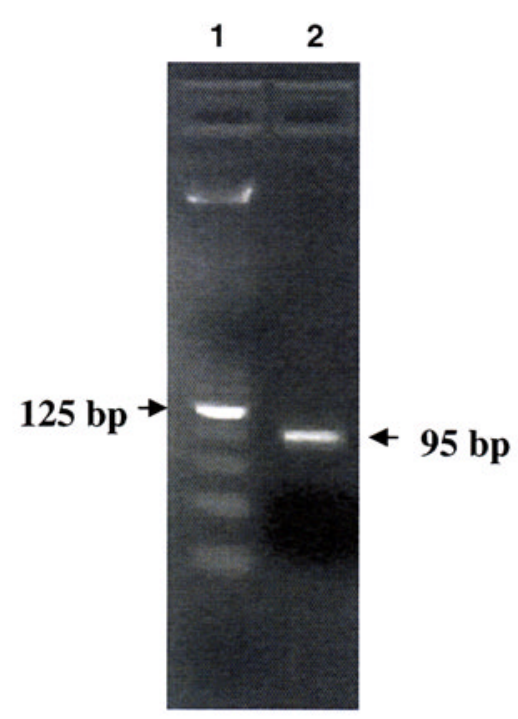

C

Previous work has shown that approximately 40,000 oligonucleotides can be attached to an individual NBC particle $\left(7 \times 10^{-11}\right.$ oligos $\left./ \mathrm{cm}^{2}\right)$ [22]. To understand the SERS signal intensity from each particle, we carefully diluted the hybridized NBC and focused onto the slide with a $100 \times$ objective using the Renishaw Raman microscope. In this way, single particles could readily be resolved and isolated for confocal SERS measurements. Under these conditions, a SERS signal could be detected from single NBC in PBS solution (Fig. 4). Using a previously determined quenching efficiency of $87 \%$ for Cy5 on an NBC [22], we can estimate that not less than $87 \%$ of the Cy 5 molecules are in very close proximity to the surface (assuming 100\% fluorescence quenching of all molecules near the surface). If the per molecule fluorescence quenching efficiency at the surface is less than $100 \%$, then a larger number of the Cy 5 molecules must be at the surface. Therefore, we estimate that between $34,800(87 \%$ of 40,000$)$ and 40,000 molecules are available to generate the SERS signal from a single particle; a number that can be readily detected. (Fig. 4).
After confirming that the observed SERS signal was created from $\mathrm{HCV}$ oligo probes on $\mathrm{NBC}$, we proceeded to hybridize the HCV target with this SERS beacon. Figure 5 shows the results of experiments where the dye-labeled HCV-metal Nanobarcode ${ }^{\mathrm{TM}}$ complex is hybridized to the $\mathrm{HCV}$ target $(10 \mu \mathrm{M})$ and two controls. PBS buffer was used as a negative control and a SARS sequence oligo $(10 \mu \mathrm{M})$ as a noncomplementary target control. Upon binding to the target DNA sequence, a large decrease in SERS signal is measured. With no target (PBS) present, the SERS signal is relatively unchanged and much larger. Finally, in the presence of a noncomplementary target, the observed change in SERS signal is much less than that seen with the correct target. (Fig. 5d).

When using a measurement technique in which a positive response is detected by a reduction or even a complete loss of signal, it is important to ensure that the loss of signal is not due to other causes. With the SERS beacon method, a potential criticism could be that the observed decrease in signal, credited to the hybridization to the target probe, 
a HCV-Cy5 NBC101010
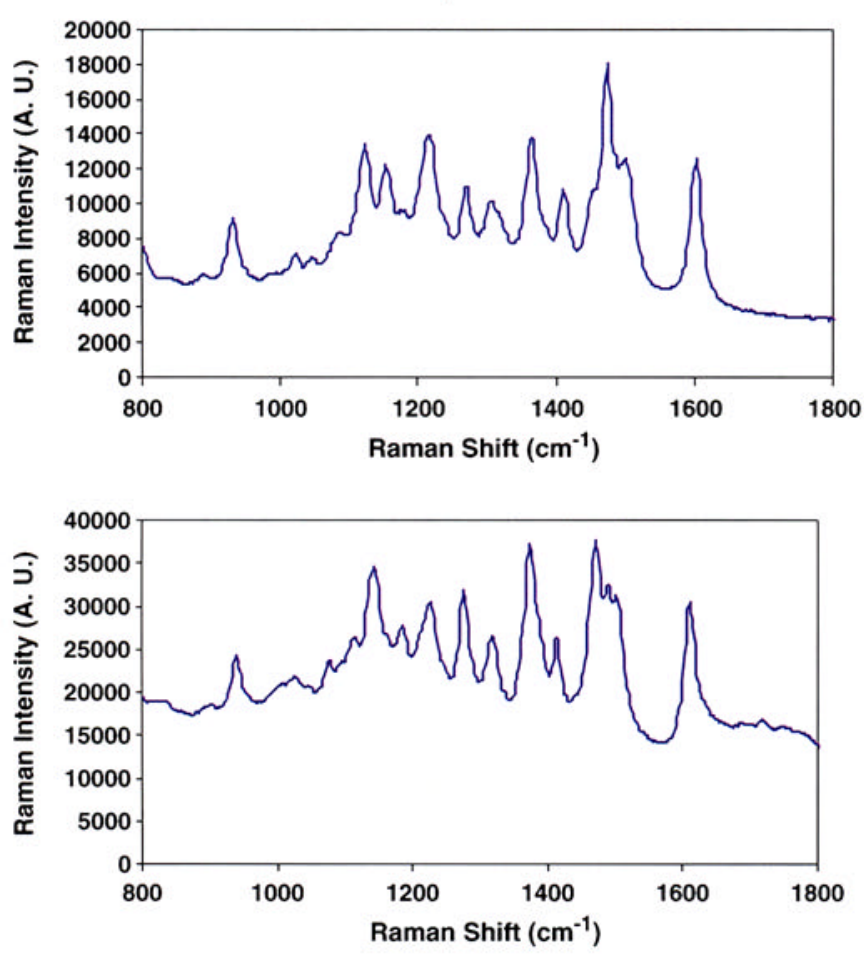

c Cy5-NBC111111 b Cy5-NBC101010
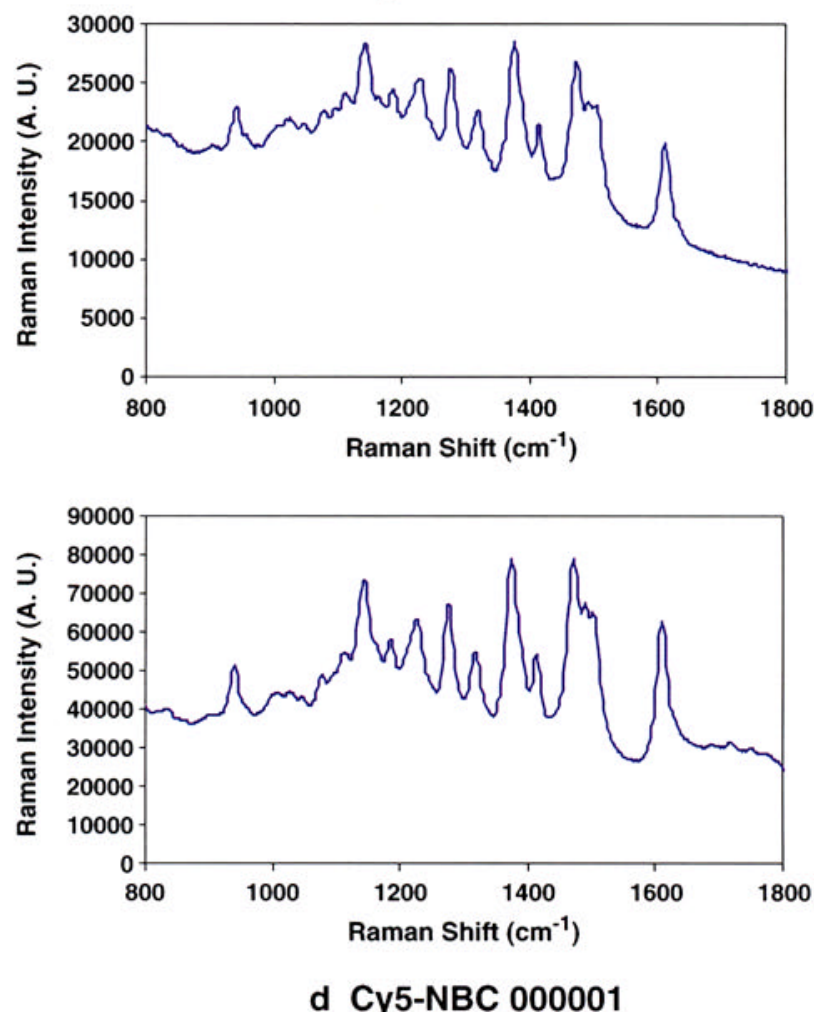

Fig. 3 SERS Spectra from different silver or gold strip pattern Nanobarcodes ${ }^{\mathrm{TM}}$ particles. a HCV-Cy5 probe conjugated to the NBC 101010 . b Cy5 mono NHS ester binding to NBC 101010. c Cy5 mono NHS ester binding to NBC111111. d Cy5 mono NHS ester binding to NBC 000001

could also be obtained by simply stripping the dye-labeled oligo from the surface of the particle. As a fluorescent molecular beacon on NBCs has already been demonstrated [22], we decided to use the fluorescence quenching response of the dye-labeled oligo to confirm that oligo remained attached to the NBC. Figures $5 \mathrm{a}, \mathrm{b}$ and $\mathrm{c}$ show the reflectance images (upper left in each group), fluorescence images (upper right), and SERS spectra (bottom) from the NBC after addition of the target. Figures $5 a$ and $c$ show that there was no fluorescence, but significant SERS after addition of either PBS or a noncomplementary target sequence. Figure $5 \mathrm{~b}$ shows that there was high fluorescence
Fig. 4 SERS spectra from single NBC. a View of NBC by $100 \times$ objective microscope. b SERS spectra from single NBC conjugated with HCV-cy5 probes under PBS solution

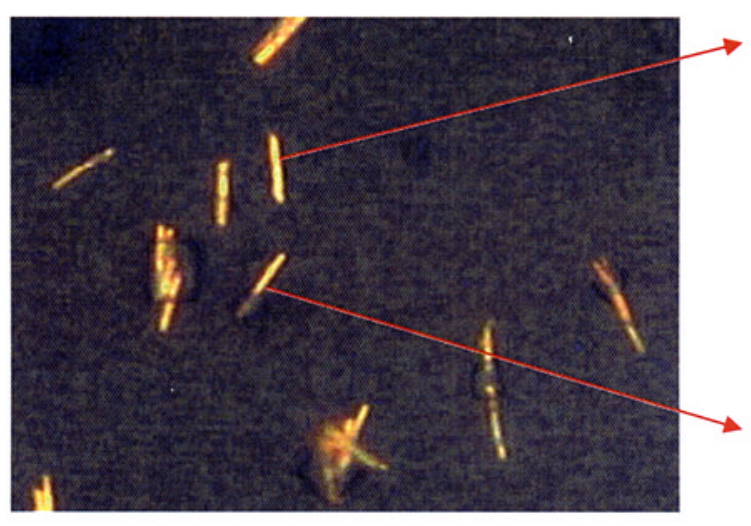

a
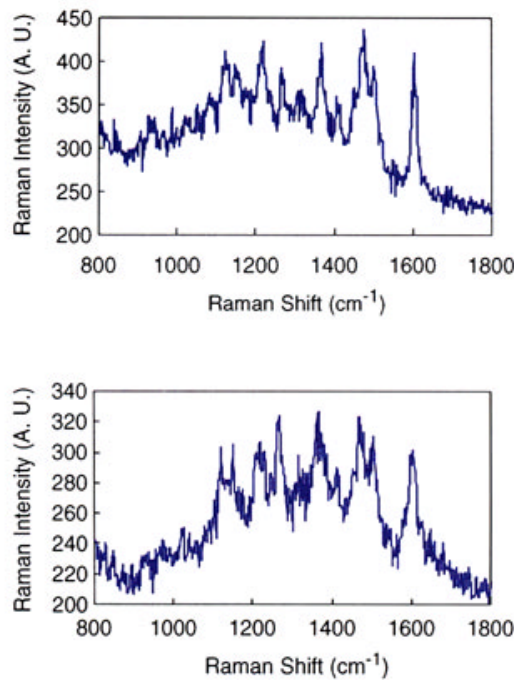

b 
a
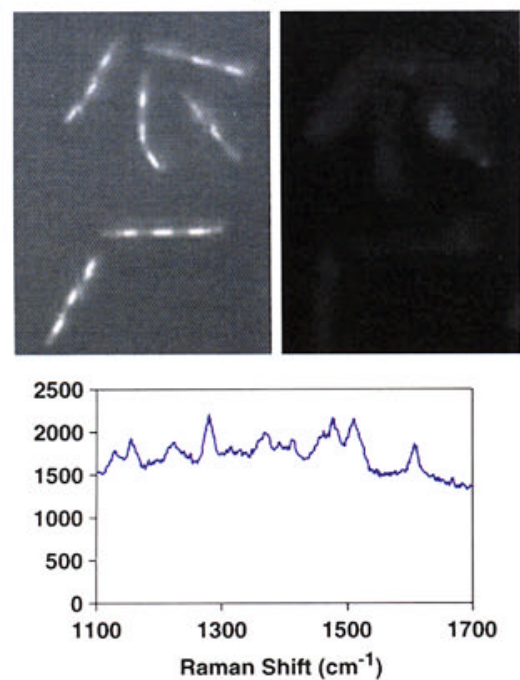

Control b
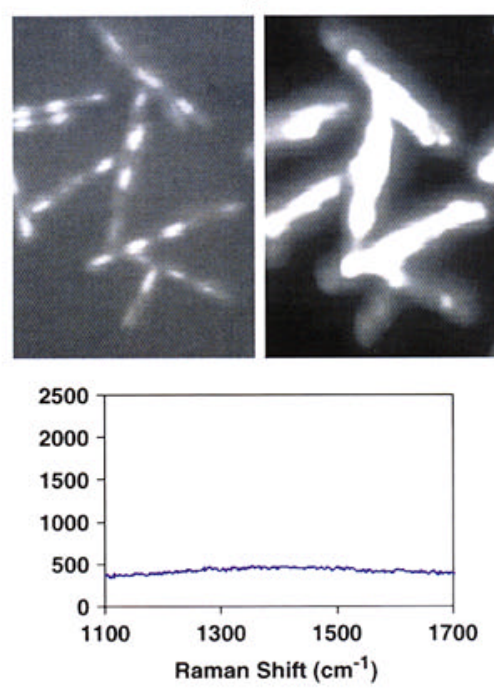

HCV c
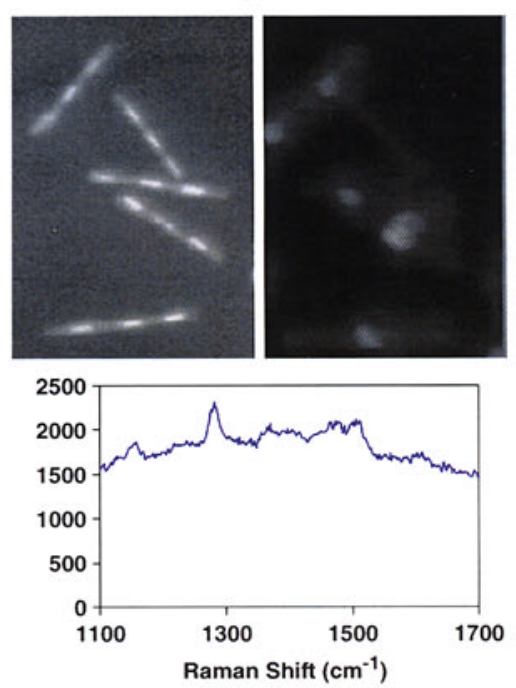

SARS

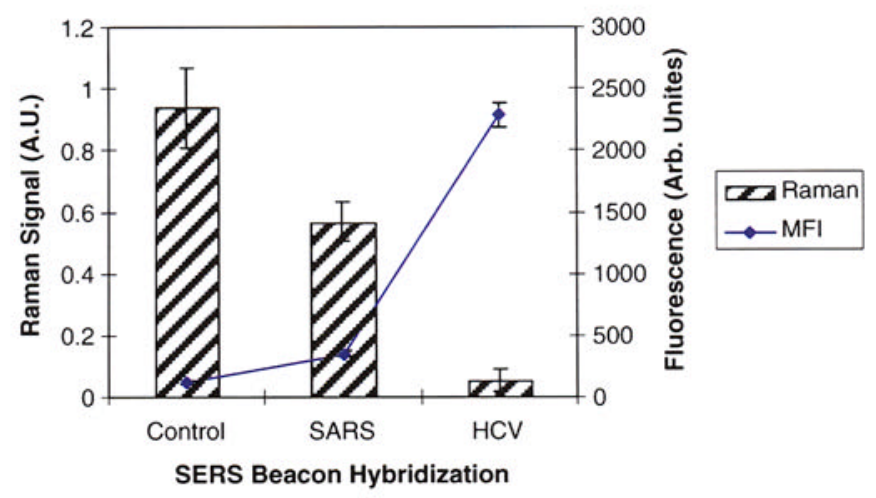

d

Fig. 5 SERS and fluorescence signal shifting when the hybridization occurs. a Negative control in the solution. Reflectance and fluorescence image pair showing location of NBCs and lack of fluorescence in the absence of target. SERS signal occurs. b HCV target presented in the reaction solution. Reflectance and fluorescence image pair showing location of NBCs and large fluorescence signal after the addition of $10 \mu \mathrm{M}$ target. SERS signal disappeared. $\mathbf{c}$ Wrong target SARS presented in the solution. Reflectance and fluorescence image pair showing location of NBCs and lack of fluorescence in the present of wrong target. SERS signal occur. d Average SERS signal was shown in column and fluorescence signal (MFI) was shown in line for these three different targets but very low SERS when the HCV target was present. This observation was also confirmed by quantitative analysis of both the Raman and fluorescence signals (Fig. 5d). The power of a dual modality SERS and fluorescent probe is shown by the fact that the fluorescence signal increased and SERS decreased when the HCV target was present. Thus, two measurements can be used to verify that the assay is giving an accurate result. This dual-detection modality could become a valuable in-built control for rapid diagnostic tests, while the NBC substrates can be used to multiplex the test and detect many targets simultaneously.

To further investigate the quantitative aspects of these SERS and fluorescent beacons, we added varying concentrations of the target to NBC-SERS beacons, analyzing samples for both SERS and fluorescence response. The data (Fig. 6) shows a strong inverse correlation between the two detection mechanisms, with the largest change in both signals occurring between 2 and $20 \mathrm{nM}$ of target oligo. The systematic anticorrelation between the SERS and fluorescence signals is convincing evidence that the system is behaving as our cartoon image implies. The clear detection of $20 \mathrm{nM}$ oligo via the SERS Beacon response also appears to be an improvement over that previously reported for a "Plasmonic Nanoprobe" system based on Ag colloid and excited at $633 \mathrm{~nm}$ [9].

To completely eliminate the SERS signal, we needed about $20 \mathrm{nM}$ target in our assay with a $50-\mu l$ reaction volume, compared to $34 \mathrm{nM}$ oligo in a $100 \mu$ l reaction 


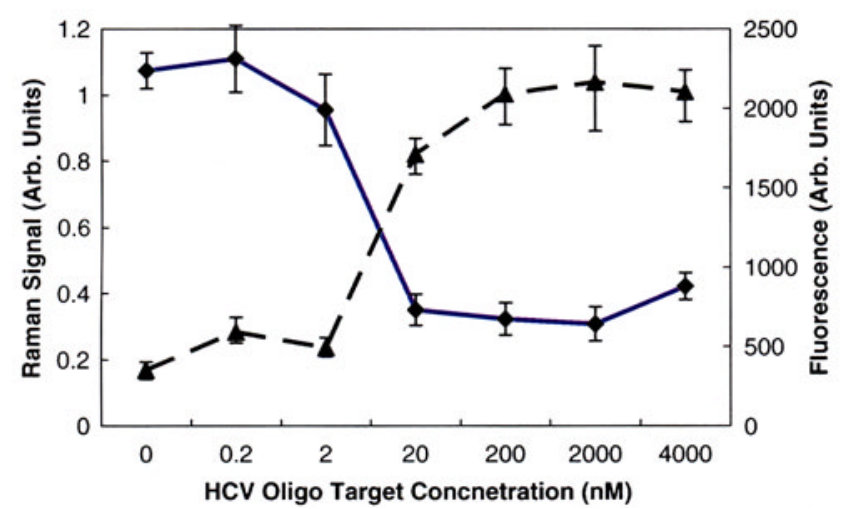

Fig. 6 Titration of SERS beacon and fluorescence molecular beacon. Solid line represents the SERS signal, while the dashed line is for the fluorescence response

volume or 2,000 $\mathrm{nM}$ with $45 \mu \mathrm{l}$ reaction volume combined with hybridization times of $2-3 \mathrm{~h}$ at room temperature in other labs $[9,10]$.

It is recognized that probe length may be a parameter for these signal shifts [22]. We compared the $34 \mathrm{mer}$ to $55 \mathrm{mer}$ probe length and found that the $34 \mathrm{mer}$ probe had a better SERS signal to noise ratio 3.1 compared to 2.7 for $55 \mathrm{mer}$ probe length, which is consistent with the fluorescent molecular beacon on NBC [22].

Finally, we demonstrated the utility of this assay using a sample closer to the real world. HCV Armored RNA ${ }^{\circledR}$ is noninfectious viral RNA, which carries the sequences of 5' UTR region of HCV and MS2 bacteriophage coat protein. This Armored $\mathrm{RNA}^{\circledR}$ is a simulated real viral RNA and is used as calibration control for clinical diagnostics [33]. We amplified the HCV RNA with RT-PCR process using HCV sequence-specific PCR primers (Fig. 2a).
Electrophoresis showed that armored HCV RNA was amplified by RT-PCR (Fig. 2c). Then, we hybridized these PCR amplicons with the SERS Beacon probe. Figure 7a shows that the SERS signal dropped when the PCR amplicon was hybridized to its probe. The SERS quenching efficiency was about $72 \%$ (Fig. $\left.7 \mathrm{~b},\left(1-\frac{\text { Signal with Target }}{\text { Signal with no Target }}\right) \times 100 \%\right)$, which is an improvement over that previously reported [9]. One of the explanations for this is the fact that the double-stranded PCR amplicon was digested before hybridization in our case (see Materials and Methods for detail) to have complementary single-stranded DNA as the target, thus, increasing the hybridization efficiency. We confirmed this by comparing the efficiency of double and single-stranded DNA using a fluorescent molecular beacon (data not shown). This system shows no SERS signal decrease using a negative control, and the fluorescence signal went up in target solution but stayed low in the negative control. This demonstrates that this system could work very well for clinical samples.

In conclusion, we have demonstrated the feasibility and usefulness of a dual system based on a SERS and fluorescent molecular beacon for detection of human viral RNA. Coupled with our previous demonstration of multiplexed fluorescence, molecular beacon for simultaneous detection of five human pathogens [22], there is a clear path to the development of a multiplexed SERS beacon by developing a Raman microscope with reflectance and fluorescence capabilities. The assay should be applicable to pathogen detection, environmental monitoring, health care and diagnostics, bio-, and chemical terrorism and in field testing for food-born pathogen. Not only is the assay label-free and multiplexed, but the dual detection mode provides internal a

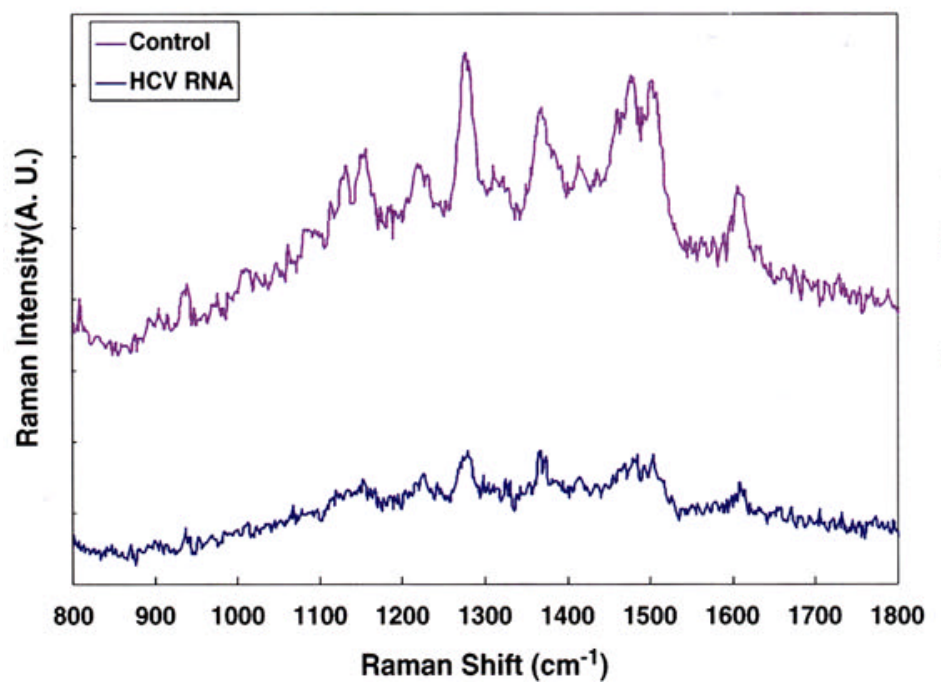

b

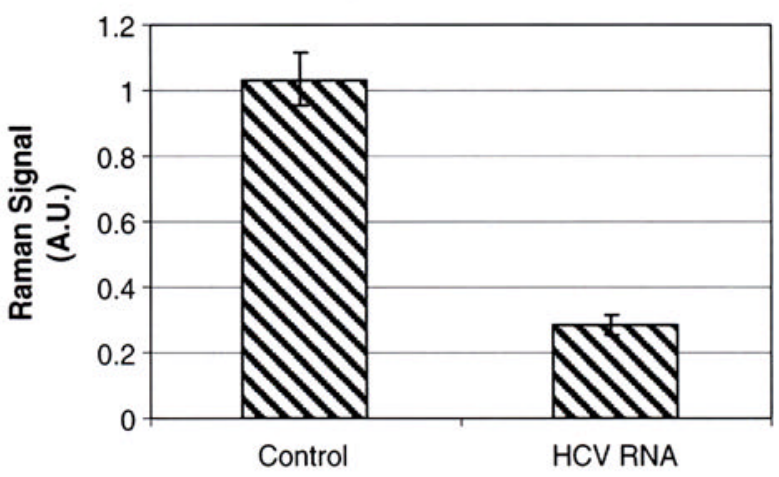

HCV RNA Target

Fig. 7 SERS Beacon for HCV RNA Detection. a Raman signal for control and HCV RNA sample after hybridization detection. b The data analysis for control and HCV RNA sample 
redundancy, making it highly attractive for rapid molecular diagnostics.

Acknowledgment The authors thank Dr. Glenn Davis for custom software programming. This work was supported by the DARPA project N66001-05-C-8040 and NIST-ATP grant number 70NANB1H3028.

\section{References}

1. Fleischman M, Hendra PJ, McQuillan AJ. Chem Phys Lett 1974;26:163-6.

2. Jeanmaire DL, Van Duyne RP. J Electroanal Chem 1977;84:1-20.

3. Cotton TM, Schulz SG, Van Duyne RP. J Am Chem Soc 1980; 102:7960-2.

4. Zhange X, Young MA, Lyandres O, Van Duyne RP. J Am Chem Soc 2005;127:4484-9.

5. Culha M, Stokes D, Allain L, Vo-Dinh T. Anal Chem 2003;75: 6196-201.

6. Shafer-Peltier KE, Laynes CL, Glucksberg M, Van Duyne RP. J Am Chem Soc 2003;125:588-93.

7. Cao YC, Jin R, Mirkin CA. Science 2002;297:1536-40

8. Faulds K, Smith WE, Graham D. Anal Chem 2004;76:412-7.

9. Wabuyele M, Vo-Dinh T. Anal Chem 2005;77:7810-5.

10. Faulds K, Fruk L, Robson DC, Enright TA, Smith E, Graham D. Faraday Discuss 2005;1-8.

11. Cao YC, Jin R, Nam J, Thaxton S, Mirkin CR. J Am Chem Soc 2003;125:14676-7.

12. Kim J-H, Kim J-S, Choi H, Lee S-M, Jun B-H, Yu K-N, et al. Anal Chem 2006;78:6967-77.
13. Mulvaney SP, Musick MD, Keating CD, Natan MJ. Langmuir 2003;19:4784-90.

14. Doering WE, Nie SM. J Phys Chem B 2002;106:311-7.

15. Nie S, Emory SR. Science 1997;275:1102-6.

16. Kneipp K, Wang Y, Kneipp H, Perelama IT, Ltzkan I, Dasari RR, et al. Phys Rev Lett 1997;78:1667-70.

17. Le Ru EC, Meyer M, Etchegoin PG. J Phys Chem 2006;110: 1944-8.

18. Tyagi S, Kramer FR. Nat Biotechnol 1996;14:303-8

19. Dubertret B, Calame M, Libchaber AJ. Nat Biotechnol 2001; 19:365-70.

20. Maxwell DJ, Taylor JR, Nie S. J Am Chem Soc 2002;124: 9606-12.

21. Du H, Disney M, Miller B, Krauss T. J Am Chem Soc 2003;125:4012-3.

22. Sha MY, Yamanaka M, Walton ID, Norton SM, Stoermer R, Keating CD, et al. Nanobiotech 2005;1:327-35.

23. Stoermer R, Cederquist K, McFarland S, Sha M, Penn S, Keating C. J Am Chem Soc 2006; 128:16892-903.

24. Lakowiez JR. Anal Biochem 2001;298:1-24.

25. Nicewarner-Pena SR, Freeman RG, Reiss BD, He L, Pena DJ, Walton ID, et al. Science 2001;294:137-41.

26. Reiss BD, Freeman RG, Walton ID, Norton SM, Smith PC, Stonas WG, et al. J Elec Chem 2002;522:95-103.

27. Walton ID, Norton SM, Balasingham A, He L, Oviso DF, Gupta JD, Raju PA, et al. Anal Chem 2002;74:2240-7.

28. Sha MY, Walton ID, Norton SM, Taylor M, Yamanka M, Natan MJ, et al. Anal Bioanal Chem 2006;384:658-66.

29. Wolkow RA, Moskovits MJ. J Chem Phys 1987;87:5858-69.

30. Campion A, Kambhampati P. Chem Soc Rev 1998;27:241-50.

31. Zuker M, Mathews DH, Turner DH. In: Barciszewski J, Clark BFC, editors. NATO ASI Series. Kluwer Academic Publishers; 1999.

32. Demers LM, Mirkin CA, Reynolds RC, Letsinger RL, Elghania, Viswanadham G. Anal Chem 2000;72:5535-41.

33. Pasloske B, Walkerpeach C, Obermodeller R, Winkler M, Dubois D. J Clin Microbiol 1998;36:3590-4. 